\title{
The practice and teaching of palpation of the head and neck: A scoping review
}

\author{
Epstein I. , Herne P., Masita S., Peisachovich E., Da Silva C., Grosman-Rimon L., Lee C., Robinson B., Eliadis M. \\ Faculty of Health, School of Nursing, York University, Toronto, Ontario, Canada
}

Received: April 29, 2017

DOI: $10.5430 /$ jnep.v7n12p20
Accepted: May 11, 2017

Online Published: July 10, 2017

\begin{abstract}
Objective: To explore how palpation of the head and neck is practiced and taught.

Methods: The scoping review methodology was guided by Arksey and O'Malley's five-stage approach. Three experienced and independent reviewers searched nine databases according to a predetermine inclusion and exclusion criteria.

Results: A total of 15 articles from medicine, chiropractic and dentistry published between 1987 and 2016 were included. Two overarching themes emerged, a Cartesian and a Pragmatic perspective in practicing and teaching palpating of the head and neck. Although both perspectives are valuable, we advocate to practice and teach palpation of head and neck from a Pragmatic perspective particularly with the increase use of ultrasound technology to detect masses. A pragmatic perspective takes into account the patient's context, the ethics of care and highlights the importance of health care providers fostering interpersonal relationships with others during physical assessment.

Conclusions: Although nursing studies were absent from this review we believe nurses play a vital role when they are aware of the Cartesian and Pragmatics perspectives when practicing and teaching head and neck palpation as part of a physical assessment. Learning how other disciplines are practicing and teaching head and neck palpation skills will improve interdisciplinary collaboration.
\end{abstract}

Key Words: Head and neck, Palpation, Practice, Teaching

\section{BACKGROUND}

Palpation is part of the overall physical assessment that is implemented by various professionals such as, medical doctors, dentists, nurses, psychiatrists, chiropractic practitioners, osteopathic medicine, physiotherapists, occupational therapists, and massage therapists to assess skin texture, to locate particular anatomy landmarks (through range of motion), and to assess tenderness. ${ }^{[1]}$ In nursing, palpation skills are used not only for assessing masses and pathology but also provide an opportunity to build a therapeutic relationship and connect with patients and their families. For example, Nishikawa and Sakakibara ${ }^{[2]}$ highlight the effects of a nursing intervention program using abdominal palpation of Leopold's maneuvers on maternal-fetal attachment. The authors highlighted that when expectant mothers were taught by nurses how to palpate their abdomen, mothers felt better connected with their unborn child.

The head and neck region contains several lymph nodes and glands, some of which have been associated with increased prevalence of cancer. ${ }^{[3]}$ Hence, accurate assessment is critical in disease detection and management. ${ }^{[3]}$ Palpation of the head and neck is taught in nursing and denture programs. If time permits, students are allowed to practice on each other

${ }^{*}$ Correspondence: Epstein I.; Email: iepstein@yorku.ca; Address: Faculty of Health, School of Nursing, York University, Toronto, Ontario, Canada. 
in the lab. Thus in this paper, we will explore how palpation of the head and neck is practiced and taught by various professions.

\section{Literature review}

Palpation is recognized as difficult to practice and teach and thus its validity is constantly being questioned. ${ }^{[4]}$ For example, several studies highlighted that it is difficult to consistently locate, visualize, sense, and describe the accurate characteristics of the masses when using palpation skills alone. ${ }^{[5-7]}$ Palpation is generally taught by teachers who first explain the anatomy and physiology of the head and neck then students practice palpation on each other while the teacher provides feedback regarding the palpation technique. ${ }^{[1]}$ However, giving students feedback may not be always accurate. ${ }^{[5,8]}$ For example, Balkissoon et al. ${ }^{[3]}$ demonstrated that during a rectal palpation exam, the teacher could not see students' hand movements or feel the appropriate pressure exerted on the, tissue and therefore, was unable to provide relevant feedback to students on their palpation performance. Other challenges that may arise from teaching and practicing palpation includes, gender sensitivity issues as students may not feel comfortable being touched by the opposite gender. ${ }^{[8,9]}$

Palpation can be used to detect swelling, pain, enlarged lymph nodes, and sites of possible infection. In addition, palpation is also used for the staging of nodal pathology; the earlier the size of a mass is detected, the more likely the cancer will be successfully treated. ${ }^{[10]}$ Moreover, cancer of the head and neck regions are more apparent to others due to the patients' disfigurement, and dysfunction. The threat to their personal identity makes early detection and treatment planning important. With an increased incidence of cancers of the head and neck, the clinician's knowledge, accurate skills and attitudes about palpation techniques are therefore critical for disease management. ${ }^{[11]}$ Missing abnormal nodes can result in delayed treatments or overestimate of enlarged nodes may lead to unnecessary workup, expense and morbidity to patients. ${ }^{[12]}$ Therefore, nurses are at the forefront of early detection as they conduct physical assessments and examination and interact with patients on a daily basis. In this paper, our purpose is to understand the practice and teaching of head and neck palpation .

\section{METHOD}

This scoping review was guided by Arksey and O'Malley's five-stage approach ${ }^{[13]}$ which include: (1) identifying the research question, (2) identifying relevant results, (3) selecting studies, (4) charting data, and (5) reporting results. We used a scoping review for three reasons. First, a scoping review allows for a better mapping of key concepts underpinning a research area and the main sources and types of evidence available. Second, a scoping review is more appropriate then a systematic review because while a systematic review focuses on a well-defined research question with a limited range of study designs, a scoping review can address broader topics and a broader range of study designs. ${ }^{[13,14]}$ Third, a scoping review is suited to our relatively broad question because it allowed for an expanded inclusion criterion to yield more research articles. For example, all study designs were eligible, including anecdotal reports (e.g., an instructor recount their personal palpation experiences). We also included studies that focused on the head or neck, head to neck and head and neck palpation by various disciplines (e.g., medicine, dentistry, chiropractic, dermatology, and athletic training education).

The first stage of the scoping review includes a clear and consistent identification of the research question. ${ }^{[13]}$ Our research question was, "What is known about the practice and teaching of the head and neck palpation skills". This broad question is open to a diversity of patient populations, conditions, practitioners and outcomes (e.g., finding a mass; hand movement; pressure exerted on skin). In the second stage, the research question was used to identify relevant studies. Our expert information specialist in consultation with two experienced nurse researchers (PH, SM) conducted a literature search independently. We searched nine electronic databases: e.g., MEDLINE, EMBASE, Cumulative Index to Nursing and Allied Health Literature (CINAHL), The Cochrane Library, PsychInfo, Social Science Abstracts, Library and Information Science Abstracts (LISA), and Education Resources Information Center (ERIC) between 1987-2016. See Table 1 for the search strategies.

Table 1. Search strategy

\begin{tabular}{lll}
\hline Palpation & Head to neck & Health practitioners \\
\hline Title-ABS-KEY (“palpation*”) & Title-ABS-KEY (“head to neck” or & Title-ABS-KEY (“nurse*” or “dentist*” or \\
Title-ABS-KEY (“touch*” or & “cranium*” or "head” or “neck” or “cervix” & "doctor*” or "physician” or "health \\
“physical assessment*”) & or “colum” or “caput” or “spinal”) & practitioner”) \\
\hline
\end{tabular}


We also retrieved articles through the Google reference search and websites of universities and institutions that teach palpation skills. The search strategy was limited to English language and text books were excluded. Arksey and O'Malley $^{[13]}$ highlighted that a quality appraisal of included literature is not required when performing a scoping review. The only quality criterion a research paper had to satisfy was to have been peer-reviewed. The third step focused on identifying the studies. The fourth stage of the scoping review included the charting of the data. ${ }^{[13]}$ Each nurse researcher analyzed articles in a table independently according to Arksey and O'Malley's ${ }^{[13]}$ recommendations. The table presented a synthesis and interpretation of the extracted data results to highlight palpation practice and teaching methods used to describe palpation skills.

\section{REPORTING RESULTS}

Arksey and O'Malley's ${ }^{[13]}$ highlight the fifth stage of the scoping review's purpose which is to report results that reflect the research question. The scoping review revealed 15 studies ranging from the years 1987 to 2016 within various disciplines (e.g., dentistry, medicine, chiropractic, athletic training, dermatology) (see Figure 1). The Nursing discipline was absent and medicine dominated from the studies included. Canada $(n=5)$; United Kingdom $(n=4)$ and United States $(n=3)$, were the countries that also dominated the review. Many were anecdotal (literature review or authors recount their personal experiences with palpations). ${ }^{[15-19]}$

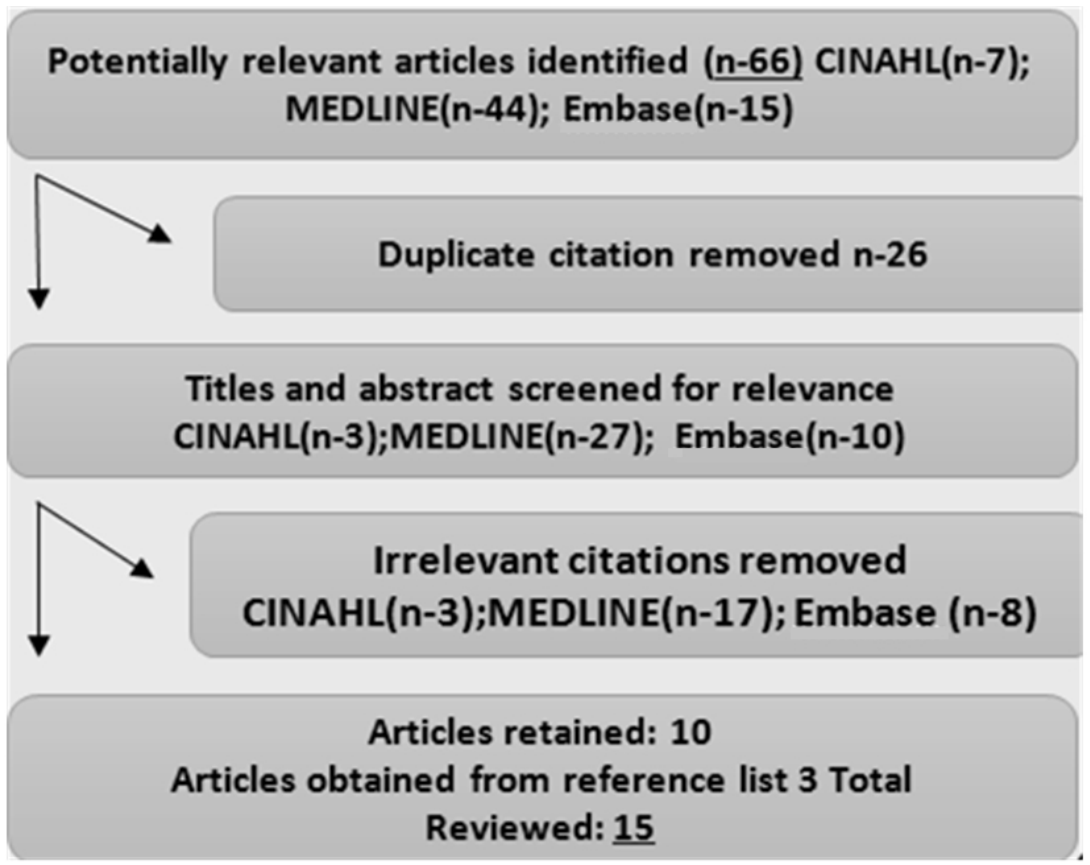

Figure 1. Scoping review process

There was a mix of methodological designs including Randomized Control Trials; ${ }^{[20]}$ correlation studies; ${ }^{[21-24]}$ mixed methods $;{ }^{[25]}$ and prospective study design. ${ }^{[23]}$ The sample size across the included studies ranged from $13^{[18]}$ to $152 .^{[19]}$ Participants were patients, medical/chiropractor students and clinicians (surgeon, dermatology).

The 15 articles included in the scoping review revealed two overarching themes: a Cartesian and pragmatic perspectives were used to describe the knowledge about the practice and teaching of palpation skills of the head and neck. ${ }^{[26]}$ During analysis of text, Miles and Huberman ${ }^{[27]}$ suggested to use concepts from the literature to further characterize overarching themes emerging from the data.

\subsection{A cartesian perspective on palpation knowledge}

The term "Cartesian perspective" (p. 228) is borrowed from Doane and Varcoe. ${ }^{[26]}$ This term has dominating influences on how we think about knowledge and knowing within health care. The Cartesian perspective emphasizes empirical studies and objective knowledge that can be separated, rationalized, logically taught and generalized. Some studies were categorized as Cartesian because of a linear trajectory of knowledge development. For example, Chaitow ${ }^{[28]}$ describes a sequential acronym of accurate palpation practice and teaching.

Therefore, a palpation skill of the head and neck regions from a Cartesian perspective focuses on the separation of the skill from its context, with a focus on being able to accurately observe and measure hence, standardizing and 
capturing the observed technique. Pringle ${ }^{[17]}$ focused on the hypothenar portion of subjects' dominant hands force exerted during palpation and professor's feedback while students (1st year chiropractor students) palpated the posterior neck. Although Pringle did not report statistically significant findings (One-way ANOVA, $p=.34$ ) between the chiropractic student groups, the students who received intermittent feedback better retained the hand technique and force exerted during palpation and thus, performed palpation techniques more accurately. Similarly, Cox ${ }^{[29]}$ used an experienced dermatologist who palpated patients $(n=16)$ while blindfolded. Cox ${ }^{[29]}$ concluded that there were statistical significance differences between patients' groups and thus (2-independent sample $t$-test, $p=.012$ ) dermatologist was accurately differentiating between skin disorder using palpation alone. Palpation is an accurate technique to distinguish blindfolded between two common types of dermatoses: atopic dermatitis and plaque psoriasis. Only Cox ${ }^{[29]}$ explicitly used a theory, whereby he focused on the dissociated sensory input concept and argued that using palpation alone, in the absence of a visual stimulus, can accurately diagnose skin conditions in the head and neck region. No further information was provided about the theory.

The studies with a Cartesian perspective regarding palpation also questioned the palpation accuracy diagnosis and thus, some advocated for the combination of palpation techniques with other tests. For example, Feinmesser et al. ${ }^{[22]}$ compared palpation to a computed tomography (CT) or ultrasound and found that while the technology was promising, it was not at the stage where it could replace palpation. With continued advances in technology, Alderson et al. ${ }^{[21]}$ advocated for physicians to rely more on ultrasound when detecting cancer in the head and neck regions as opposed to palpation alone. Similarly, Haberal et al. ${ }^{[23]}$ argued that while palpation is a valuable skill, CT paired with ultrasonography (USG) provided accurate and reliable results when combined together. Hoang et al. ${ }^{[30]}$ advocated for a systematic approach in assessing the cervical lymph nodes highlighting the use of CT and MRI, while de-emphasizing the need for palpation as smaller nodes can harbor metastases and can be difficult to palpate (p. 17).

Through a Cartesian perspective, assumptions and facts about palpation practice are unchangeable regardless of context. ${ }^{[26]}$ However, several authors who focused on the accuracy of palpation questioned palpation skills in relation to its context (i.e., who performed the palpation). There were differences between expert (more than two years' clinical experience) and novice (students) practitioners in relation to locating pathological masses during palpation. ${ }^{[21,24,31]}$ For example, Alderson et al. ${ }^{[26]}$ used a partial trainer model to test the link between practitioner experience and their diagnostic accuracy of cancer in the head to neck. They found that practitioner experience and confidence was not correlated with practitioner abilities to accurately differentiate particularly small nodes in the head and neck. Additionally, a study by $\mathrm{Xu}$ et al. ${ }^{[24]}$ added that although there were no differences between students and experts palpating pathological masses that were larger than $2 \mathrm{~cm}$, more experienced surgeons located accurately smaller masses (less $1.5 \mathrm{~cm}$ ) than students. Chaitow ${ }^{[28]}$ suggested similar viewpoints and ascertained that experienced practitioners are more focused and will record more accurate significant findings because they understand what is meaningful, rather than being overwhelmed by data (p. 131). These articles were categorized as examples of a Cartesian perspective of palpation because they focused on accuracy of palpation techniques.

\subsection{A pragmatic perspective to palpation knowledge}

On the other hand, a pragmatic perspective on palpation views palpation knowledge in its changing context. ${ }^{[26]}$ For the practitioner who adapts a pragmatic perspective on palpation knowledge, their own body is an important source of knowledge. For example, the practitioner's emotions and feelings are as important, as are the physical objective findings, such as, signs and symptoms and hence palpation is a complex process. Chinnah et al. ${ }^{[25]}$ reported that students' own feelings of comfort touching their peers during palpation affected their palpation practices and skill development.

We categorized the aforementioned studies under pragmatic approaches because the main objective was not to focus on the accuracy of palpation skill, but rather to highlight the relationship of the people and context while practicing and learning palpation skills. For example, teaching palpation with hands-on practice is more valuable than memorizing particular hand movements and its related sequence. Punj et al. ${ }^{[19]}$ reported statistical significance (ANOVA; $p=.001$ ) between students' abilities to palpate various skin disorders (seborrheic keratosis; squamous cell and basal cell carcinoma) during "hands on practice" (p. 806), after they reviewed case studies as opposed to passively learning content online. On the other hand, Rafai et al. ${ }^{[20]}$ found a statistical significant difference on the Objective Structured Clinical Examination (OSCE) results ( $T$-test; $p=.0007$ ) when clinical practice was combined with palpation e-learning training. Furthermore, Leppington et al. ${ }^{[18]}$ added that "vertical integration" (p.51), the idea of weaving palpation skill teaching throughout the curriculum rather than at one moment in time is vital. They reported that $17 / 20$ participants $(85.7 \%)$ had to use palpation skills during their internship.

The value of using simulation to recreate context prior to 
performing palpation with real patients was highlighted by several authors. Eberman and Finn ${ }^{[16]}$ anecdotally reported the need to teach palpation gradually. They explained that "learning palpation is like learning to read" (p. 171) and argued that gradually introducing students to a simulation of a physical examination (palpation) prior to interaction with real patients would enhance students learning. Chinnah, De Bere and Collett ${ }^{[25]}$ conducted a focus group and submitted questionnaires to 3rd-4th year medical students. They reported that when students performed palpation with a peer/life model, as opposed to using cadavers, students felt more comfortable connecting to others with the therapeutic touch of palpation. However, there were no statistical significance differences between using peer students and a standardized patient (human actor). Aubin, Gagnon, and Morin ${ }^{[15]}$ focused on a cyclical process of teaching and practicing palpation skills. Their 7-step PALPATE approach invites students and faculty to focus more on their interpersonal rather than the outcome (i.e., finding a mass). The authors argued that palpation skills require a different type of knowledge, motor, perceptual and therapeutic attitude. For them "palpation is an open-ended task that seeking an undefined solution" (p. 66). ${ }^{[15]}$

Most studies that used pragmatic perspectives regarding head and neck palpation were guided by theory that exemplified the complexity of context. For example, Aubin, Gagnon, and Morin ${ }^{[15]}$ used cased load theory and motor skill learning theory. These theories highlighted the cognitive and motor complexity required from practitioners during palpations. Chinnah, De Bere and Collett ${ }^{[25]}$ used patient-centered care concept focusing on the empathy that developed between the patient and practitioner during palpation. Eberman and Finn ${ }^{[16]}$ used critical thinking emphasizing the gradual learning and practicing of palpation; shifting from knowledge to evaluating and synthesizing complex mass location and patients' circumstances. Raffi et al. ${ }^{[20]}$ used experiential learning theory to emphasize the concept of peer teachingdoing during head and neck palpation.

\section{Discussion}

The 15 reviewed articles revealed two overarching themes that there are Pragmatic and Cartesian perspectives on the practice and teaching of head and neck palpation skills. A pragmatic perspective highlighted the relationship between the practitioner and the context when performing palpation skills. To be able to palpate effectively the practitioner must learn to feel, and be attuned with self and others as this sensing is an important source of knowledge. Similarly, Doane and Varcoe ${ }^{[26]}$ highlight the skill of conscious participation when nurses relate to self and others within a pragmatic perspective where "conscious participation involves using our senses within a social position" (p. 163). ${ }^{[26]}$ Limited studies in this scoping review discussed the ethics and patients' social positioning within performing palpation skills. For example, asking a patient's permission to touch them or palpate as well as providing culturally sensitive care when engaging in palpating clients. Only Chinnah, De Bere and Collette $^{[25]}$ briefly discussed culture within patient-centered care concept. Coldicott, Pope, and Roberts ${ }^{[32]}$ recognize that the ability to communicate sensitively is crucial during palpation. Indeed, the patient's perspective on what is or is not an intimate examination must be central during physical exam of the head and neck. ${ }^{[33]}$ Several authors reviewed obtained Research Ethics Board approval for their study ${ }^{[18,24,25,29]}$ yet many did not. Miser ${ }^{[34]}$ distinguishes between curricular evaluation and education research and highlights the need to involve earlier in the process the ethics review board.

Thus, a pragmatic perspective highlights that palpation is not made of isolated skills but must be taught within context. For example, during an ethics course palpation skills and ethics can be emphasized. Thus, Doane and Varcoe ${ }^{[26]}$ highlighted that a pragmatic approach helps the practitioner to be in synch with self and other during assessment as "being in sync involves paying attention to how you are as you relate to others" (p. 347). As well, Leppington et al. ${ }^{[18]}$ acknowledges that weaving palpation skills through curriculum in simulation case studies through "vertical integration" will highlight the different context students can practice palpation skills (p. 51). Recognizing the gradual teaching from understanding to synthesizing and analyzing the information received by palpation in critical ways is key.

A pragmatic approach to palpation skills is fundamental to nursing. Yet in this scoping review, we could not locate nursing studies focusing on the practice and teaching of head and neck palpation skills. Nurses value the skill of palpation as its value goes beyond the identification of a pathogen but also includes interpersonal interactions. ${ }^{[22]}$ In nursing undergraduate education students are not tested on head and neck palpation skills in the OSCE but rather on deep abdomen palpation. Thus perhaps head and neck palpation is not seen as valuable enough in nursing curricular and belongs to other specialties. Other disciplines that practice and teach palpations skills, including massage therapists, physiotherapists, and respiratory therapy were absent as well. Nevertheless, this scoping review revealed that most studies were dominated by physicians who view palpation from a Cartesian perspective.

Those viewing palpation from a Cartesian approach focused on capturing palpation skills and measuring it; hence, gener- 
alizing the objectification of palpation skills. This is understandable as early detection of mass correlates to increased survival rates. ${ }^{[35]}$ Thus, using a high fidelity task trainer was advocated by many authors in this scoping review. ${ }^{[21,24,26]}$ Similarly, other authors advocated for the standardization of palpation skills and the use of task trainers as a safer teaching approach. ${ }^{[8,9]}$ Additional authors outside the studies included in this scoping review focused on the standardization of pressure/forces exerted on the skin and hand movements. ${ }^{[36-38]}$ Some of the authors ${ }^{[17,21-23,28,30]}$ who followed the Cartesian perspective of the head and neck palpation skills argued that palpation does not render consistent and accurate results and suggested abandoning the skill of palpation and relying on more accurate technology such as, ultrasound since it is available, accurate, and safe. Developing countries and remote areas of low- and middle-income countries where assessment by health care providers and resources are often scarce, and in some cases lacking, there is an increase in ultrasound services provided by unregulated individuals. ${ }^{[39]}$ Thus, in some circumstances, this may be acceptable but we fear that as technology replaces healthcare practitioners and their use of palpation skills, abandoning its practice may become widespread. We are faced with the following question, "Should educators abandon the teaching of palpation in the health science curriculum where technology, such as ultrasound is readily available?"

Paley et al., ${ }^{[40]}$ a group of emergency physicians, argued that the increased use of ultrasound due to advances in technology can be costly and may compromise a patients' quality of care because if clinicians can do good assessment not everyone will require an ultrasound. Thus, we are proposing to bring head and neck palpation teaching back not only to nursing but other disciplines. Malone ${ }^{[41]}$ named this "distal nursing" (p. 2321) and linked it to ethics. She argued that the physical exams are opportunities to be with our patients (p. 2322) and abandoning it may not only have an impact on patient's outcomes, but also on our moral decision making, as we care less for patients in a proximal way. Proximal care with patients facilitates clinician's conscious participation and "being with and doing with". (p. 287) ${ }^{[26]}$ This scoping review has revealed that palpation skills are practiced and taught by diverse disciplines with a Cartesian or Pragmatic perspective. Both perspectives are valuable, yet we advocate that the pragmatic perspective and practice in teaching palpation skills which takes into account the patient's context. Viewing palpation from a pragmatic approach highlights the value of human consciousness and the importance of fostering interpersonal relationships with our patients. ${ }^{[26]}$ This scoping review also highlights the diverse disciplines that practice and teach head and neck palpation. Learning what other disciplines are doing is a step toward improving interdisciplinary collaboration, safe and a best practices approach to care. ${ }^{[42-44]}$ Furthermore, future research should focus on interdisciplinary collaboration using task trainers and simulation to teach head and neck palpation in its diverse contexts.

\section{CONFLICTS OF INTEREST Disclosure}

The authors declare that there is no conflict of interest.

\section{REFERENCES}

[1] Jarvis C. Physical examination \& health assessment. (7th ed.) St. Louis, Mo: Elsevier. The Joanna Briggs Institute (JBI). Reviewers' Manual 2015 Methodology for JBI Scoping Reviews. 2015.

[2] Nishikawa M, Sakakibara H. Effect of nursing intervention program using abdominal palpation of Leopold's maneuvers on maternalfetal attachment. Reproductive Health. 2013; 10(12): 1-7. Available from: http://www.reproductive-health-journal.com/con tect/10/1/12 https://doi.org/10.1186/1742-4755-10-1 2

[3] Shetty D, Jayade BV, Joshi SK, et al. Accuracy of palpation, ultrasonography, and computed tomography in the evaluation of metastatic cervical lymph nodes in head and neck cancer. Indian Journal of Dentistry. 2015; 6(3): 121-124. https ://doi .org/10 $.4103 / 0975-962 X .163032$

[4] Liebenson C, Lewit K. Palpation's reliability: a question of science vs. art? Journal of Body Works and Movement and Therapy. 2013; 7(1): 46-48. https://doi .org/10.1016/S1360-8592(02)00106-7

[5] Balkissoon R, Blossfield K, Salud L, et al. Lost in translation: unfolding medical students' misconceptions of how to perform a clinical digital rectal examination. The American Journal of Surgery. 2009;
197(4): 525-32. https://doi.org/10.1016/j.amjsurg. 2008 .11 .025

[6] Low-Beer N, Kinnison N, Baillie S, et al. Hidden practice revealed: using task analysis and novel simulator design to evaluate the teaching of digital rectal examination. The American Journal of Surgery. 2011; 201: 46-53. PMid:21167365 https ://doi.org/10.1016/ j.amjsurg. 2010.09.004

[7] Laufer S, Cohen E, Maag A, et al. Multimodality Approach to Classifying Hand Utilization for the Clinical Breast Examination. Studies in Health Technology and Informatics. 2014; 196: 238-244. PMid:24732514

[8] Pugh C. Application of National Testing Standards to SimulationBased Assessments of Clinical Palpation Skills. Military Medicine International Journal of AMSUS. 2013; 178(10): 55-63. PMid:24084306 https://doi.org/10.7205/MILMED-D-13-0 0215

[9] Pugh CM, Blossfield Iannitelli K, Rooney D, et al. Use of MannequinBased Simulation to Decrease Student Anxiety Prior to Interacting With Male Teaching Associates, Teaching And Learning In Medicine. 2012; 24(2). https://doi.org/10.1080/10401334 .2012 .664534 
[10] Lyman GH, Giuliano AE, Somerfield MR, et al. American Society of Clinical Oncology Guideline Recommendations for Sentinel Lymph Node Biopsy in Early-Stage Breast Cancer. Journal of Clinical Oncology. 2005; 23(30): 7703-7720. https://doi.org/10.1200/ JC0. 2005.08.001

[11] Shetty D, Jayade BV, Joshi SK, et al. Accuracy of palpation, ultrasonography, and computed tomography in the evaluation of metastatic cervical lymph nodes in head and neck cancer. Indian Journal of Dentistry. 2015; 6(3): 121-124. https://doi .org/10 $.4103 / 0975-962 \mathrm{X} .163032$

[12] Kau R, Alexiou C, Stimmer H, et al. Diagnostic procedures for detection of lymph nodes metastases in cancer of the larynx. Oral Journal of Otorhinolaryngology Related Spectrum. 2000; 62: 199-203. https://doi.org/10.1159/000027746

[13] Arksey H, O'Malley L. Scoping studies: Towards a methodological framework. International Journal Social Research Methodology. 2005; 8(1): 19-32. http://dx.doi.org/10.1080/13645570320 00119616

[14] Pham MT, Rajic A, Greig JD, et al. A Scoping review of scoping reviews: advancing the approach and enhancing the consistency. Research Synthesis Methods. 2014; 5: 371-385. https: //doi.org/10.1002/jrsm.1123

[15] Aubin A, Gagnon K, Morin C. The seven-step palpation method: A proposal to improve palpation skills. International Journal of Osteopathic Medicine. 2014; 17(1): 66-72. https://doi.org/10.101 6/j.ijosm.2013.02.001

[16] Eberman LE, Finn ME. Enhancing Clinical Evaluation Skills: Palpation as the Principle Skill, Athletic Training Education Journal. 2010; 5(4): 170-175.

[17] Pringle RK. Guidance hypothesis with verbal feedback in learning a palpation skill. Journal of Manipulative and Physiological Therapeutics. 2004; 27(1): 36-42. https://doi.org/10.1016/j.jmpt.2 003.11 .004

[18] Leppington C, Gleberzon B, Fortunato L, et al. Degree of Vertical Integration Between the Undergraduate Program and Clinical Internship With Respect to Cervical and Cranial Diagnostic and Therapeutic Procedures Taught at the Canadian Memorial Chiropractic College. The Journal of Chiropractic Education. 2012; 26(1): 51-61. PMid:22778531 https://doi.org/10.7899/1042-5055-26.1. 51

[19] Punj P, Devitt PG, Coventry BJ, et al. Palpation as a useful diagnostic tool for skin lesions. Journal of Plastic, Reconstructive \& Aesthetic Surgery. 2014; 67(6): 804-807. https://doi.org/10.1016/j. bjps.2014.02.009

[20] Rafai N, Lemos M, Kennes LN, et al. Anatomy meets dentistry! Linking anatomy and clinical practice in the preclinical dental curriculum. Medical Education. 2016; 16: 305.

[21] Alderson DJ, Jones TM, White SJ, et al. Observer error in the assessment of nodal disease in head and neck cancer. Journal of the Specialties of the Head and Neck. 2001; 739-743.

[22] Feinmesser R, Freeman JL, Noyet AM, et al. Metastatic Neck Disease; A Clinical/Radiographic/Pathologic Correlative Study Archives of Otolaryngological Head and Neck Surgery. 1987; 113: 1307-1310. PMid:3675897 https://doi .org/10.1001/archot ol.1987.01860120053007

[23] Haberal I, Celik H, Gocmen H, et al. What is important in the evaluation of metastatic lymph nodes in head and neck cancer: Palpation, ultrasonography, or computed tomography? OtolaryngologyHead and Neck Surgery. February 2004; 197-201. PMid:14990916 https://doi.org/10.1016/j.otohns.2003.08.025
[24] Xu JJ, Campbell G, Alsaffar H, et al. Lymphadenopathy: Defining a palpable lymph node. Journal of the Specialties of the Head and Neck. 2015; 177-181. https://doi.org/10.1002/hed. 23578

[25] Chinnah TI, De Bere SR, Collett T. Students' views on the impact of peer physical examination and palpation as a pedagogic tool for teaching and learning living human anatomy. Medical Teacher. 2011; 33(1): e27-e36. https://doi.org/10.3109/0142159X.2011.530313

[26] Doane GH, Varcoe C. How to nurse: Relational inquiry with individuals and families in changing health and health care contexts. 2015.

[27] Miles MB, Huberman AM. Qualitative data analysis: An expanded sourcebook. Thousand Oaks: Sage Publications; 1994.

[28] Chaitow L. The ARTT of palpation? Journal of Bodywork and Movement Therapies. 2012; 16(2): 129-131. https://doi.org/10.1 016/j.jbmt. 2012.01.018

[29] Cox N. A literally blinded trial of palpation in dermatologic diagnosis. Journal of American Academy of Dermatology. 2007; 56(6): 949-951. https://doi.org/10.1016/j.jaad.2007.01.044

[30] Hoang J, Vanka J, Ludwig B, et al. Evaluation of Cervical Lymph Nodes in Head and Neck Cancer with CT and MRI: Tips, Traps, And a Systematic Approach American Journal of Roentgenology. 2013.

[31] Xu JJ, Fung K, Glicksman JD, et al. Development of a tissue-mimicking neck model for medical education. Journal of Otolaryngology-Head \& Neck Surgery. 2012; 41(2).

[32] Coldicott Y, Pope C, Roberts C. The ethics of intimate examinations -teaching tomorrow's doctors. British Medical Journal. 2003; 326: 97-101. PMid:12521977 https://doi .org/10.1136/bmj . 326 . 7380.97

[33] Schenck D. Ethical Considerations in the Treatment of Head and Neck Cancer. Cancer Control. 2002. Available from: https://moffitt.org/File\%20Library/Main\%20Nav/Rese arch $\% 20$ and $\% 20$ Clinical $\% 20$ Trials/Cancer\%20Control $\% 20$ Journal/v9n5/410.pdf

[34] Miser W. Educational Research-To IRB, or Not to IRB? Family Medicine. 2005; 37(3): 168-173.

[35] Kolb T, Lichy J, Newhouse J. Comparison of the Performance of Screening Mammography, Physical Examination, and Breast US and Evaluation of Factors that Influence Them: An Analysis of 27,825 Patient Evaluations, Radiology. 2002; 225: 1. Available from: http://pubs.rsna.org/doi/abs/10.1148/radi ol .2251011667? journalCode=radiology PMid:12355001 ht tps://doi.org/10.1148/radiol.2251011667

[36] Keating J, Matyas T, Bach T. The effects of training on physical therapists ability to apply specific forces of palpation. Physical Therapist. 1993; 73(1): 38-46. https://doi.org/10.1093/ptj/73.1.38

[37] Lederman SJ, Klatzky RL. Hand movements: a window into haptic object recognition. Cognitive Psychology. 1987; 19(3): 342-68. https://doi.org/10.1016/0010-0285(87)90008-9

[38] Salud LH, Pugh CM. Use of sensor technology to explore the science of touch. Studies in Health Technology and Informatics. 2011; 163: 542-8. PMid:21335853

[39] Sippel S, Muruganandan K, Levine A, et al. Use of Ultrasound in the developing world, International Journal of Emergency Medicine. 2011; 4: 72. https://doi .org/10.1186/1865-1380-4-72

[40] Paley L, Zornitzki T, Cohen J, et al. Utility of clinical examination in the diagnosis of emergency department patients admitted to the department of medicine of an academic hospital. Archives Internal Medicine. 2011; 171(15): 1394-1396.

[41] Malone RE. Distal nursing, Social Science \& Medicine. 2003; 56(11): 2317-2326. https://doi.org/10.1016/S0277-9536(02) 002 $30-7$ 
[42] Axelsson SB, Axelsson R. From territoriality to altruism in interprofessional collaboration and leadership. Journal of Interprofessional Care. 2009; 23(4): 320-330. PMid:19517284 https: //doi.org/10.1080/13561820902921811

[43] Bokhour B. Communication in interdisciplinary team meeting: What are we talking about? Journal of Interprofessional Care. 2006; 20(4):
349-363. PMid:16905484 https://doi.org/10.1080/135618 20600727205

[44] Reese LE, Sontage MA. Successful interpersonal collaboration on the hospice team. Health and Social Work. 2001; 26(3): 167-175. PMid:11531192 https://doi .org/10.1093/hsw/26.3.167 